\title{
Pathologies des vésicules séminales
}

\author{
Jean HERMABESSIERE \\ Pôle Santé République, Clermont-Ferrand
}

\section{RESUME}

Le regain d'intérêts pour les troubles de la fertilité masculine permet de redécouvrir les vésicules séminales. Leur pathologie a été bien étudiée dans le premier quart du XXe siècle. La douleur est évocatrice. II s'agit de douleurs avec projection périnéale et irradiation le long du trajet du cordon spermatique. Les sécrétions peuvent être modifiées et prendre un aspect purulent ou sanglant.

Les examens complémentaires font appel à l'étude du sperme et en particulier la spermoculture. Quant à l'imagerie, l'échographie endorectale reste le premier examen.

Les différentes pathologies des vésicules séminales sont l'infection, les tumeurs malignes ou bénignes, les malformations, les lithiases.

Mots clés : pathologie des vésicules séminales, imagerie des vésicules séminales

\section{INTRODUCTION}

La pathologie des vésicules séminales est actuellement un peu oubliée. II y a eu pourtant un rapport présenté à la Société Belge d'Urologie en 1921 et, un rapport au Congrès Français d'urologie en 1929 (par Minet). Nous pensons que c'est parce qu'à l'ère des antibiotiques on ne voit plus d'infection chronique des vésicules séminales et, que grâce à ces médicaments la prostatite ne devient plus une prostato-vésiculite. Le regain d'intérêt pour les troubles de la fertilité masculine permet cependant de revoir les troubles de cet organe. On peut les classer en i) pathologie infectieuse, ii) pathologie tumorale, iii) pathologie malformative, iii) pathologies rares (lithiase et calcifications, lésions amyloïdes, fistules).

\section{SIGNES CLINIQUES ET EXAMENS COMPLEMENTAIRES DE LA PATHOLOGIE DES VESICULES SEMINALES [8]}

\section{Douleurs}

Les douleurs sont des symptômes évocateurs : douleurs locales avec ténesme vésical et projection périnéale. Les irradiations sont funiculaires (le long du trajet du cordon), il peut même exister des coliques spermatiques bien décrites par Doussot en 1932.

La caractéristique de la douleur est son trajet de la bourse à la fosse iliaque pouvant faire évoquer une appendicite si elle siège à droite.

Les douleurs peuvent être également d'irradiation basse vers les aines, la face interne des cuisses ou vers le haut: région sacrée et lombaire. Ces douleurs sont déclenchées ou augmentées par la défécation et, parfois la miction, la symptomatologie urinaire peut être trompeuse.

II y a modification des sécrétions : pollution purulente ou sanglante, écoulement après une selle ou un toucher rectal, pyospermie clinique ou biologique, hémospermie. Il y a également des symptômes sexuels : érections douloureuses, pollution douloureuse, éjaculation prématurée (évocatrice si secondaire) puis impuissance.

Cette symptomatologie peut s'accompagner de signes généraux qui sont identiques à la prostatite aigüe qui est souvent associée et, il peut même y avoir une rétention d'urines.

Correspondance :

Dr Jean HERMABESSIERE - Pôle Santé République, 99 Avenue de la République, 63023 Clermont-Ferrand Cedex Tel 04.73.42.39.51 - Fax 04.73.91.63.11 -

Email jhermabessiere@centrerepublique-chtm.com 


\section{Examens}

Les examens complémentaires portent sur l'urine et le sperme. Etude bactériologique du premier jet d'urines couplée à la spermoculture. Ces examens permettent de rechercher des germes et de pratiquer l'antibiogramme.

Le spermogramme est modifié (les vésicules séminales sécrètent environ $60 \%$ du volume de l'éjaculât). Lorsqu'il y a un défaut de sécrétion, il s'agit d'hypospermie. Lorsqu'il y a une hypersécrétion, il y a hyperspermie. Le $\mathrm{pH}$ du sperme est alcalin, le fructose peut être modifié, c'est le meilleur marqueur des vésicules séminales.

La spermoculture sera pratiquée au besoin sur la deuxième partie de l'éjaculât. Elle permet la numération des leurcocytes, des hématies, des germes et l'antibiogramme.

L'examen clinique est toujours difficile, il s'agit de palper les vésicules séminales qui se situent au-dessus et latéralement par rapport à la prostate. On perçoit assez souvent une tumeur souple et sensible. Parfois, il existe un véritable signe de la sonnette : la palpation par le doigt rectal déclenche une douleur qui suit le trajet du cordon.

\section{L'image des vésicules séminales $[10,16]$}

Elle comporte l'échographie endo-rectale qui est un examen simple qui permet de bien visualiser la taille des vésicules et l'existance éventuelle de concrétions. Le scanner et l'IRM sont des examens qui sont intéressants pour rechercher l'envahissement dans les pathologies tumorales.

Enfin, la vésiculo-déférentographie reste le meilleur examen pour étudier la taille, l'aspect anatomique et l'état de la muqueuse des vésicules séminales. Il est cependant invasif, car réalisé en salle d'opération, avec abord chirurgical des déférents. Après un abandon total, cet examen reprend un regain d'intérêt.

\section{LES PATHOLOGIES DES VESICULES SEMINALES}

\section{L'infection des vésicules séminales}

Appelée aussi spermato-cystite, elle est souvent associée à une prostatite etou à une épididymite $[2,11,12,18,20]$.

a) l'infection aigue se caractérise par des douleurs et des signes généraux : une douleur à l'examen, au toucher rectal et une spermoculture positive, avec pyospermie et hémospermie. Elle est rarement rencontrée de nos jours.

b) l'infection sub-aigue et chronique est assez fréquente dans les cas de stérilité. La symptomatologie est faite de pesanteur, d'hémospermie, avec retentissement sur la fertilité : asthénospermie, flagelles anormaux, bactériospermie, polynucléaires altérés dans le sperme. Les germes retrouvés peuvent être des germes banaux en nombre supérieur à $10^{4}$, des germes spécifiques : tuberculose, brucellose (rares actuellement) ou les germes intra-cellulaires: mycoplasmes et chlamydiae. On peut également trouver des parasites comme la bilharziose. Cette dernière pathologie est souvent diagnostiquée sur l'examen vésical qui montre les œufs sous muqueux.

\section{La pathologie tumorale $[3,4,9,13]$}

\section{a) les tumeurs malignes}

Elles sont rarement primitives. Elles sont alors des adénocarcinomes ou exceptionnellement des sarcomes. Le diagnostic est difficile car ces tumeurs évoluent lentement. II n'y a pas de symptomatologie particulière. Le diagnostic repose sur le scanner ou l'IRM qui permettent de voir que la tumeur est vésiculaire sans lésion prostatique. La biopsie fait souvent le diagnostic.

Les tumeurs malignes secondaires sont beaucoup plus fréquentes. II peut s'agir d'un envahissement par contiguïté d'un cancer vésical ou prostatique ou digestif, ou même d'un processus métastatique. II peut également s'agir d'une localisation d'une hémopathie.

\section{b) les tumeurs bénignes [1, 6, 15, 19]}

Lorsqu'elles sont volumineuses, elles peuvent donner une symptomatologie à la miction ou à la défécation, mais elles sont le plus souvent asymptomatiques. II peut s'agir de kystes, d'adénomes, de polypes, de fibromes, de cystadénomes, de neurofibrome. Les plus fréquentes de ces tumeurs bénignes restent les kystes ou hydrospermatocystes. Ces lésions sont alors dues soit à une obstruction du canal éjaculateur soit à une lithiase vésiculaire ou à des séquelles d'infection. Au toucher rectal, on peut sentir une masse susprostatique qui est souple. La ponction ramène un liquide renfermant des spermatozoïdes, des leucocytes et des hématies. L'échographie est le premier temps de l'imagerie qui peut être complété par un scanner ou une IRM.

\section{Les malformations $[14,17]$}

Elles sont en rapport avec un développement anormal du canal de Wolff : vésicules petites, atrophiques ou absentes. Ces anomalies sont de découverte fortuite. On peut observer une agénésie unilatérale, simple ou associée à d'autres anomalies génitales. L'absence d'une ou des deux vésicules séminales sans anomalie urinaire est évocatrice de mucoviscidose.

Une vésicule peut être absente par fusion des deux vésicules, avec un seul canal excréteur et un abouchement ectopique dans l'utricule. II y a également des dysplasies vésiculaires : vésicules hypoplasiques ou vésicules hyperplasiques (vésicules pseudo-kystiques) multi-cloisonnées, volumineuses refoulant parfois la vessie.

II peut égaleemnt y avoir des kystes congénitaux associés à d'autres localisations kystiques. Ces kystes ne sont ni wolfiens, ni mullériens. Ils ne sont pas non plus des diverticules des canaux éjaculateurs et ne sont pas non plus des kystes prostatiques.

Une dernière malformation est représentée par l'abouchement ectopique de l'uretère dans la vésicule séminale [7]. Cette malformation s'accompagne souvent d'une agénésie rénale. Les signes de découverte sont une infection, des 


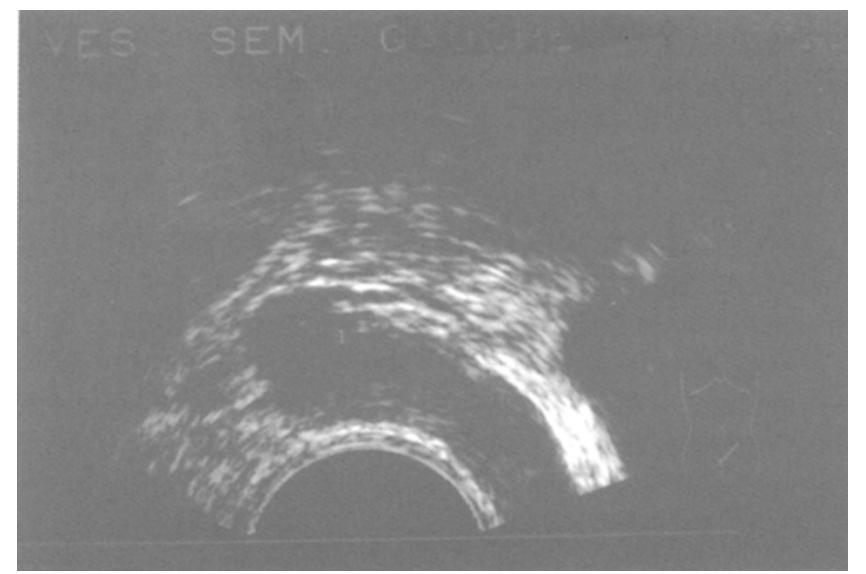

Figure 1 : A propos d'un cas clinique de dilatation d'une vésicule séminale : échographie vésicule séminale droite dilatée.

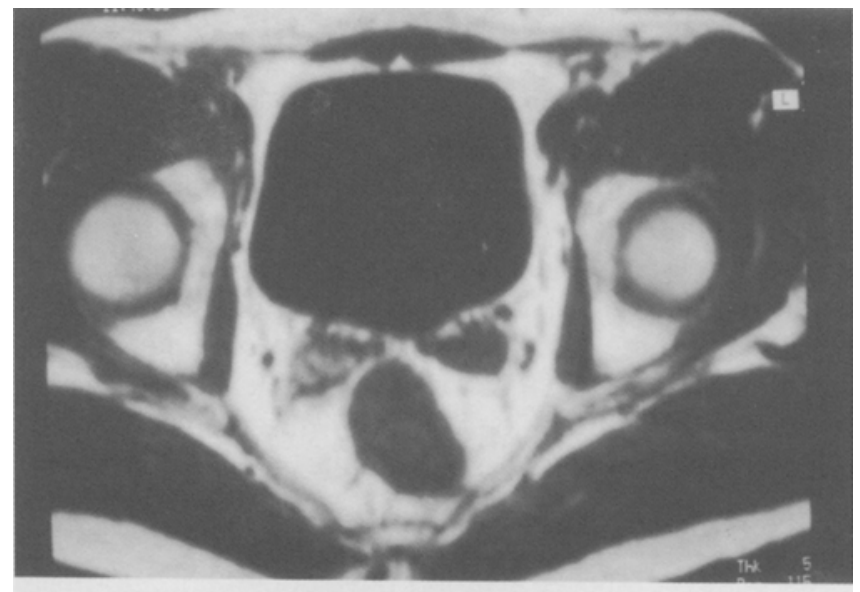

Figure 3

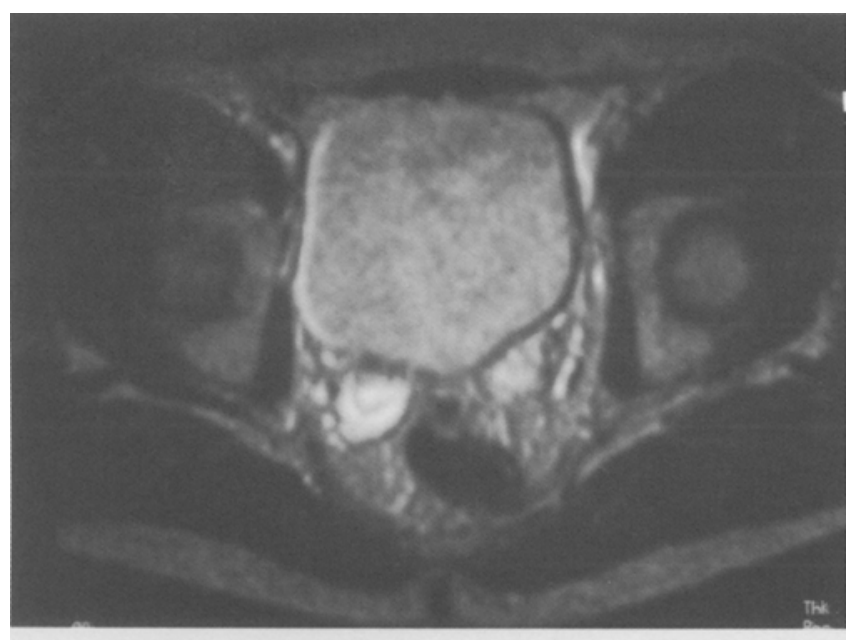

Figure 5

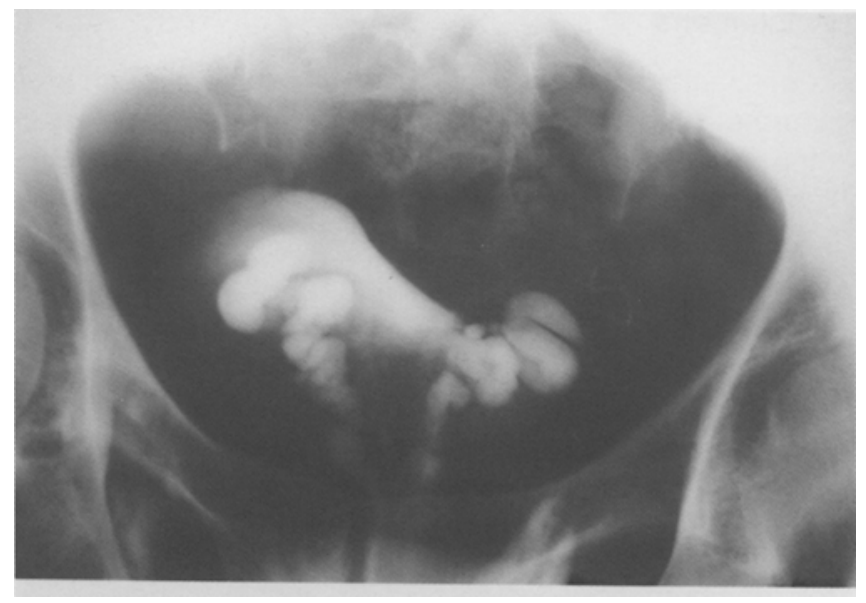

Figure 2 : A propos d'un cas clinique de dilatation d'une vésicule séminale : vésiculo-déférentographie. Les deux vésicules sont globuleuses. La droite est un peu dilatée.

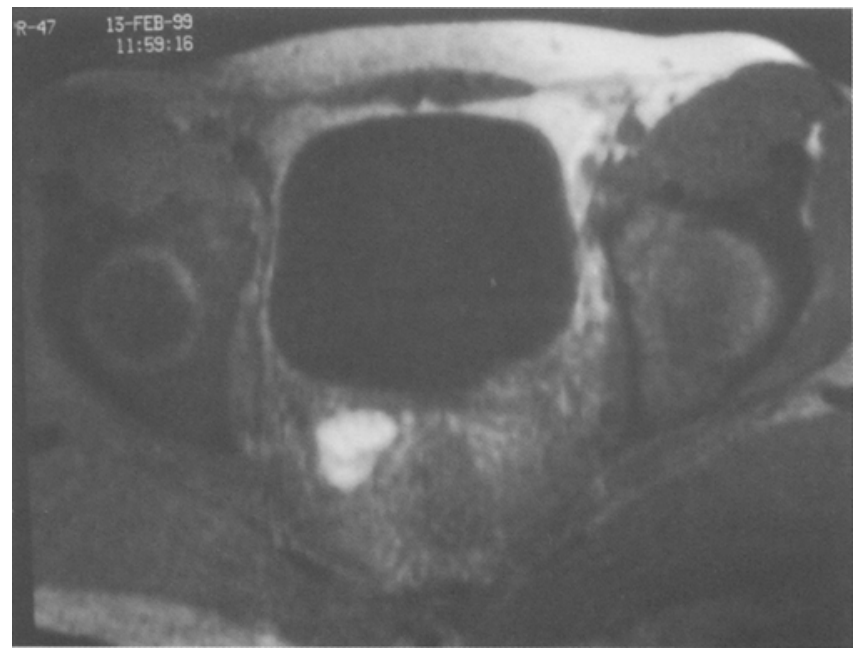

Figure 4

Figure 3, 4, 5 : A propos d'un cas clinique de dilatation d'une vésicule séminale : résonance magnétique. Visualisation des vésicules séminales avant et après Gadolinium. 
douleurs hautes ou basses. L'imagerie permet le diagnostic. L'exérèse peut maintenant être faite par coelioscopie.

4. Les autres pathologies $[5,8]$

\section{a) les lithiases}

Elles sont de deux types soit en association avec une lithiase rénale avec abouchement utétéral ectopique dans la vésicule séminale, ce qui est rare, soit possiblement dues à un reflux d'urines dans les canaux éjaculateurs. Les signes révélateurs sont l'hématurie, les douleurs, les troubles mictionnels et sexuels, l'hémospermie. Le diagnostic est fait sur l'arbre urinaire sans préparation si elles sont radioopaques ou à l'échographie lorsqu'elles ne le sont pas.

\section{b) les calcifications vésiculaires}

Il existe des calcifications vésiculaires, soit qu'il s'agisse d'un calcul volumineux, soit qu'il s'agisse d'une calcification post-tuberculeuse.

L'amylose des vésicules séminales est peu fréquente. II s'agit d'une tumeur vésiculaire dont le diagnostic est fait par la biopsie.

\section{c) les fistules}

Elles sont le plus souvent iatrogènes, après adénomectomie par voie haute, plus rarement après résection endoscopique d'un adénome prostatique. Elles sont bien visualisées par l'urétro-cystographie rétrograde et mictionnelle. II existe alors une symptomatologie de reflux urnaire dans la vésicule séminale, qui se voit également lorsqu'il y a eu résection des canaux éjaculateurs.

\section{CONCLUSION}

La pathologie des vésicules séminales, organes qui fabriquent les trois cinquièmes du volume de l'éjaculât, est à dominante infectieuse, mais il existe beaucoup d'autres lésions, qui bien que rares, doivent être présentes à l'esprit de l'andrologue.

\section{REFERENCES}

1. BASCHINSKY D.Y. et al. : Seminal vesical cystadenoma. Urology, 1998, $51: 840-845$

2. BAUER K.M. : Inflammatory diseases of the seminal vesicles, their diagnosis and therapy. Urologue, 1962, $1: 245-252$.

3. BERGER ANDREAS P. : Primary rhabdomyosarcoma of the seminal vesicie. J. Urol., 2002, $168: 643$.

4. BUCK C., SHAW W.R.E. : Primary tumor of the retrovesical region with special reference to mesenchymal tumeurs of the seminale vesicle. Br. J. Urol. 1972, $44: 47$.

5. CHAUVIN E. : Maladie des vésicules séminales. Paris, Editions Médicales N. Maloine, 1930.

6. CORDONNIER $\mathrm{C}$. : Les tumeurs rares de la prostate ou des vésicules séminales. Ana-path., 1996, $16: 37-40$.

7. DASSOULI B. : Le kyste de la vésicule séminale associé à une agénésie rénale ipsilatérale. Progr. Urol., 2001, 11 : 685-689.

8. GUELLIOT O. : Des vésicules séminales. Thèse de Médecine, Paris, 1985.

9. HADJU 1., FARUQUE A. : Adenocarcinome of the seminal vesicle. J. Urol., 1968, 99 : 798.
10. HERMABESSIERE J., ROCHE G., VIALLET J.F., USSEIL C.F.: Vésiculographie chez l'homme stérile. Revue Franç. Gynéco-obst., 1977.

11. HERMABESSIERE J., SIROT J., BOUCHER D., CLUZEL R. : Critères cliniques et bactériologiques de l'infection génitale chez l'homme stérile. Revue Franç. Gynéco-obst., 1977.

12. HERMABESSIERE J.: Les spermatocytes. In : Arvis G., ed. Andrologie Tomme II. Paris, Maloine, 1989.

13. JEFFERSON T. : Schwannoma of the seminal vesicle. ConMed., 2002, $66: 259-260$.

14. KING B.E. et al. : Congenital cystic disease of the seminal vesicle. Radiology, 1991, $207:$ 178-180.

15. LATCHAMSETY-KALYAN C. et al. : Schwannoma of a seminal vesicle. Urology, 2002, $60: 515$.

16. LITTRYP P., LEE F., MCLEARY R., KUMASAKA G.H. Transrectal US of the seminal vesicles and ejaculatory duct : clinical correlation. Radiology, 1988, $168: 625$.

17. MEGE J.L. et al. : Malformations of wolffian duct derived male internal geniral organs (epididymis, vas deferens, seminal vesicles, ejaculatory ducts). Progr. Urol., 1997, $7: 262-269$.

18. MOBLEY D.F. : Semen culture in the diagnostic of bacterial prostates. Urology, $1975,114: 83-85$.

19. MORIN G. et al. : Tumeur fibreuse solitaire des vésicules séminales : à propos d'un cas. Progr. Urol., 1998, 8 : 92-94.

20. PRADIER M. : Des spermatocystites chroniques non tuberculeuses et du traitement. Thèse de Médecine, 1922.

Manuscrit reçu : novembre 2005 ; accepté janvier 2006.

\section{ABSTRACT}

Seminal vesicle diseases

\section{Jean HERMABESSIERE}

The renewed interest in male infertility has led to a more detailed investigation of the seminal vesicles. Seminal vesicle disease were well studied in the first quarter of the 20th century. Symptoms suggestive of seminal vesicle disease are pain with perineal projection and irradiation along the spermatic cord. Secretions can be modified with a blood-stained or purulent appearance.

Complementary investigations comprise semen studies and particularly semen culture. Transrectal ultrasound remains the first-line imaging examination.

The various seminal vesicle diseases are infection, benign or malignant tumours, malformations, and stones.

Key-Words: seminal vesicle disease, seminal vesicle imaging 